\title{
Placenta acreta en aborto tardío con posterior histerectomía
}

\section{Placenta accreta in late term abortion with subsequent hysterectomy}

\author{
Gustavo Federico Gómez Quintero', Andrés Alberto Bahamón Mendoza', Fidel Ernesto \\ Ferreira Narváez ${ }^{2}$
}

\begin{abstract}
Resumen
La placenta acreta en el embarazo temprano es una entidad poco frecuente pero debe ser considerada una complicación severa del embarazo que puede ser asociada con hemorragia masiva anteparto e intraparto pudiendo potencialmente comprometer la vida. En este estudio se presenta una paciente con cesárea anterior y ecografía del segundo trimestre que reporta placenta de implantación anterior baja, que se presenta como un aborto inevitable de 20 semanas de gestación, posterior a maduración cervical y expulsión fetal con retención placentaria y hemorragia masiva, requiriendo histerectomía subtotal de emergencia con una recuperación posterior a la cirugía adecuada. El estudio de patología reportó placenta acreta. La placentación anormal en el embarazo temprano ha tenido un incremento marcado debido al aumento en la tasa de cesáreas en las últimas décadas. El objetivo principal de reportar este caso es enfatizar en el reconocimiento temprano de las alteraciones de placentación en el primer y segundo trimestre del embarazo, en aquellas pacientes con factores de riesgo es necesario una evaluación detallada en búsqueda de acretismo, con el fin de poder anticipar complicaciones derivadas de esta patología y así poder impactar positivamente en la morbilidad y mortalidad materna asociada.
\end{abstract}

Palabras clave: placenta acreta, hemorragia, histerectomía, aborto, cesárea.

\begin{abstract}
Placenta accreta in early pregnancy is not a common condition but one that should be considered as a severe complication of pregnancy, associated with massive antepartum or intrapartum haemorrhage which can be life-threatening. This study presents the case of a patient with a previous caesarean birth and a second semester scan showing placenta accreta, regarded as an inevitable cause for abortion at 20 weeks of pregnancy. Following cervical ripening and expulsion of the foetus with placental retention and massive bleeding, an emergency subtotal hysterectomy was required with recovery after surgery. The pathology study showed placenta accreta. Abnormal placentation in early pregnancy has seen a marked increase due to the increase in caesareans in recent decades. The main objective of studying this case is to emphasise the importance of early recognition of changes of placentation in the first and second trimesters of pregnancy. In patients with risk factors, a thorough assessment looking for placenta accreta is necessary in order to be able to anticipate complications associated with this pathology and thus have a positive impact on the associated maternal morbidity and mortality.
\end{abstract}

Key words: placenta accreta, haemorrhage, hysterectomy, abortion, caesarean.

1. MD. Residente de Ginecología y Obstetricia, Universidad Surcolombiana, Hospital Universitario de Neiva, Colombia.

2. MD. Especialista Ginecología y Obstetricia. Especialista en Medicina Materno Fetal, Universidad Surcolombiana, Hospital Universitario de Neiva, Colombia.

Correspondencia: Gustavo Federico Gómez Quintero: Correo electrónico: drfedegomez@ yahoo.es

Recibido: 25/11/2013-Revisado: 10/11/2014-Aceptado: 15/12/2015 


\section{Introducción}

La placenta acreta ocurre cuando toda o parte de la placenta se implanta de manera anormal al miometrio ${ }^{1}$. Se han definido tres grados de placentación anormal de acuerdo a la profundidad de la invasión demostrado por histopatología:

Acreta: Las vellosidades coriales están en contacto con el miometrio.

Increta: Las vellosidades coriales invaden dentro del miometrio.

Percreta: Las vellosidades coriales invaden la serosa uterina.

En aquellos pacientes en los que se les hace el diagnóstico histológico de placentación anormal, $81.6 \%$ de los casos fueron placenta acreta, $11.8 \%$ de los casos fueron placenta Increta y $6.6 \%$ fueron placenta percreta en 1 estudio observacional $^{2}$.

La placenta acreta es considerada una complicación severa del embarazo que puede estar asociada con hemorragia masiva anteparto e intraparto y potencialmente comprometer la vida ${ }^{3}$. Cerca del $51 \%$ de las histerectomías de emergencias son a causa de placenta acreta, la morbilidad materna ha sido reportada hasta en un $60 \%$ y la mortalidad hasta de un $7 \%$ en las mujeres con placenta acreta, la incidencia de las complicaciones perinatales son incrementadas principalmente por la prematurez ${ }^{4}$.

La placenta acreta puede ser un problema letal cuando la encontramos durante la dilatación, evacuación y curetaje posterior a una pérdida del embarazo en el primer o segundo trimestre que conduce a una hemorragia obstétrica masiva minutos después de iniciado el procedimiento. El siguiente caso nos ejemplifica la necesidad de sospechar y evaluar agresivamente los defectos de placentación en pacientes con historia de cesárea anterior o curetaje que puedan aumentar la morbilidad y la mortalidad tanto materna como fetal y nos lleve a una actuación médica oportuna a favor de las pacientes ${ }^{7}$.

\section{Presentación del caso}

Paciente de 35 años (G2 C1) con embarazo de 20 semanas por ecografía de primer trimestre, quien consulta por cuadro clínico de siete horas de amniorrea abundante sin otra sintomatología. Tiene ecografía obstétrica de las 19 semanas que reporta placenta corporal anterior baja.

Al examen físico: altura uterina de $18 \mathrm{~cm}, \mathrm{FCF} 132$ por minuto, al tacto vaginal dilatación de $4 \mathrm{~cm}$, borramiento de $80 \%$, líquido amniótico claro. La paciente es hospitalizada para manejo de aborto inevitable.

La conducta seguida consistió en maduración cervical con Misoprostol $200 \mathrm{mcg}$ endovaginal, produciéndose 6 horas posterior a la aplicación la expulsión del feto con retención de placenta, sangrado aproximado de $1000 \mathrm{cc}$ e inestabilidad hemodinámica. Se sospechó acretismo placentario y la paciente es traslada a salas de cirugía previa reserva de unidad de cuidados intensivos y componentes sanguíneos.
Se procedió a realizar histerectomía abdominal subtotal de emergencia. Se evidenció útero con acretismo e infiltración hasta serosa de vejiga y solución de continuidad en segmento uterino, y después se realizó reanimación con cristaloides.

La paciente fue trasladada a unidad de cuidados intensivos obstétrica para monitoreo hemodinámico donde evolucionó satisfactoriamente y fue enviada a hospitalización al día siguiente donde permaneció aproximadamente dos días, egresando sin complicaciones.

Se recibió estudio de patología reportando descripción macroscópica, en formol se recibe útero sin anexos que pesa 360 gr y mide 11.5 x 9 × $5.5 \mathrm{cms}$, de color pardo rosado con áreas violáceas superficie lisa y consistencia firme. Al corte la pared miometrial mide $3.5 \mathrm{cms}$, la cavidad endometrial se encuentra ocupada por tejido hemorrágico de aspecto placentario que compromete el miometrio sin romper la serosa (Figura 1).

Descripción microscópica: El endometrio presenta cambios deciduales en toda su extensión. El miometrio se encuentra infiltrado en su tercio superior por tejido placentario sin compromiso de la serosa (Figura 2).

\section{Discusión}

La placenta acreta es una entidad prácticamente nueva, fue descrita por primera vez en el siglo 20. En 1937 Irving y Hertig definieron placenta Acreta como "la adherencia placentaria anormal, ya sea en su totalidad o en parte, posterior al nacimiento a la pared uterina". El hecho de que la placenta acreta no fuera descrita por los anatomistas y patólogos de los siglos XVIII y XIX quienes describieron la mayoría de las lesiones que conocemos actualmente, sugiere que la condición no existía o era demasiado rara por lo cual no fue diagnosticada antes de $1930^{7}$.

La patogénesis exacta de la placenta acreta es aún desconocida. Dentro de las posibles etiologías se incluyen factores mecánicos como daño de la decidua basal causado por trauma local de la pared uterina, factores biológicos como respuesta materna anormal a la invasión trofoblástica o una combinación de ambos procesos ${ }^{4}$.

La placenta acreta era una entidad poco frecuente entre 1930 a 1950, con una incidencia de uno en 30.000 nacimientos, sin embargo esta frecuencia aumentó a uno en 19.000 nacimientos entre 1950 a 1960, pero en 1980 la tasa incrementó a uno en 7.000, se ha documentado un incremento en la frecuencia de hasta cuatro veces entre 1994 a 2002. En la actualidad muchos investigadores citan la frecuencia de placenta acreta tan alta como tres por cada 1000 nacimientos en la última década ${ }^{5}$, este incremento en la incidencia de placenta acreta se ha visto relacionado directamente con el aumento en la tasa de cesáreas, la cual en Estados Unidos era de 5.5\% en 1970 , de $24.7 \%$ en 1988 y de $31.8 \%$ en 2007 , con una tendencia a incrementarse aún más, llegando a reportarse en algunos estudios tasas de cesáreas tan altas como 53\% ${ }^{6}$.

El término general "placenta acreta" es usado para referirse a los diferentes grados de adhesión placentaria anormal 


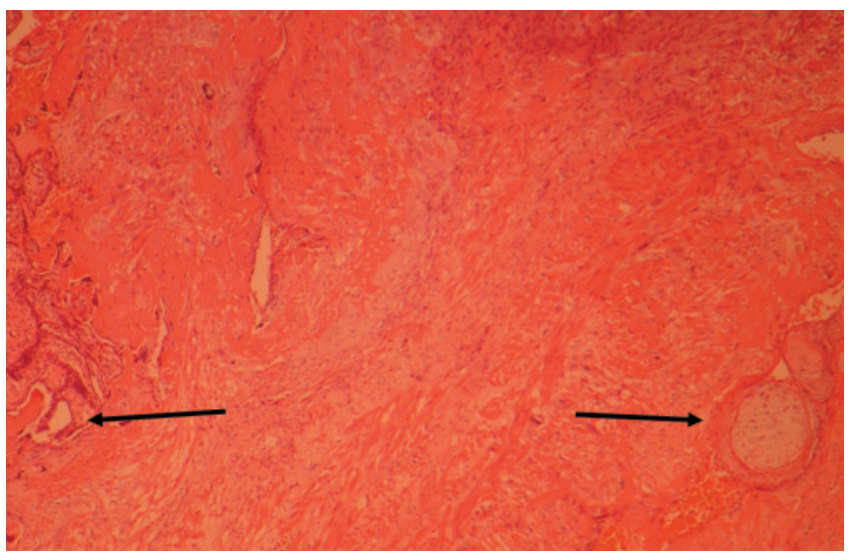

Figura 1. En microscopio de luz corte de hematoxilina eosina con aumento de 100x, al lado izquierdo se ven vellosidades coriales en contacto con el miometrio sin decidua interpuesta, al lado derecho se observa vellosidad corial profundamente insertada dentro del miometrio.

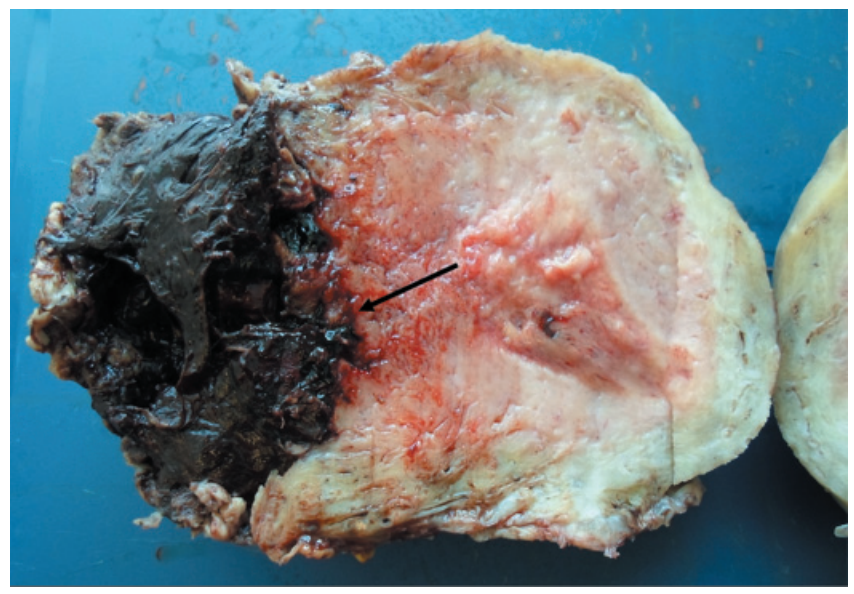

Figura 2. Útero puerperio temprano, con extensa zona hemorrágica en el segmento y en la transición al canal endocervical. Se observa el fondo de la cavidad uterina sin tejido placentario.

a la pared uterina, los cuales son caracterizados por invasión del trofoblasto dentro de miometrio en ausencia de decidua basal y de la banda fibrinoide de Nitabuch. La placenta increta y percreta son entidades raras y representan menos del $20 \%$ de los casos de la placenta Acreta $^{2,7}$, el incremento de esta entidad en las últimas décadas probablemente es secundario al aumento en la tasa de cesáreas, en los Estados Unidos fue reportada en un 5\% de todos los nacimientos en 1970 subiendo a un $32.8 \%$ en $2010^{8,9}$.

Es muy importante para el Obstetra identificar factores de riesgo para desarrollar placenta acreta en mujeres con daño miometrial por una cesárea anterior y un posterior embarazo con placenta previa, así como la implantación de placenta sobre la cicatriz uterina previa. Silver y cols reportaron en estas mujeres, el riesgo para la primera, segunda, tercera, cuarta y quinta o más cesáreas, en 3.3\%, 11\%, 40\%, 61\% y $67 \%$ respectivamente ${ }^{10}$. Una sola cesárea anterior sin placenta previa es por sí sola un factor de riesgo que incrementa la incidencia de placenta acreta asociado al incremento en el número de las cesáreas anteriores: $0.2 \%$ para la primera, $2.1 \%$ con la cuarta y mayor de $6.7 \%$ con la sexta o más, adicionalmente la placenta previa por sí sola, sin cirugía uterina previa, es asociada en $1 \%$ a $4 \%$ como factor de riesgo para placenta acreta ${ }^{10,11}$. Otros factores de riesgo también han sido reportados como la edad materna avanzada que incrementa el riesgo por cada año por encima de los 20 años, así como cirugías uterinas previas, entre ellas la miomectomia, ablación endometrial, dilatación y curetaje que pueden llevar a daño endometrial severo como el síndrome de Asherman $^{3,4,11,12}$. Revisando la literatura se encuentran pocos casos reportados de placenta acreta en el primer o segundo trimestre del embarazo. Rashbaum, et al. describió una prevalencia de $0,04 \%$ de placenta acreta encontrada durante la dilatación y evacuación en el segundo trimestre. Ellos revisaron siete casos de placenta acreta encontrados durante la dilatación y evacuación en el segundo trimestre, de los cuales todos requirieron histerectomía ${ }^{13}$.

La histerectomía obstétrica de emergencia ha sido realizada tradicionalmente como medida para salvar la vida en el manejo de hemorragia uterina no controlada. La hemorragia posterior al parto sigue siendo en Colombia la principal causa de morbilidad y mortalidad materna, la histerectomía obstétrica de emergencia parece ir en aumento en $0.026 \%$ en países desarrollados y $0.28 \%$ en países en vía de desarrollo ${ }^{14}$. Lone $\mathrm{F}$, et al. reportaron en su estudio, una tasa de realización de histerectomía de emergencia de $73.1 \%$ como manejo de hemorragia obstétrica intratable posterior a una cesárea, de las cuales todas las mujeres tenían al menos una cesárea anterior. A pesar de agentes farmacológicos y nuevas técnicas quirúrgicas para el control de la hemorragia posterior al parto se ha encontrado que no existe reducción en la prevalencia de la histerectomía obstétrica de emergencia. La cesárea anterior con presencia de alteraciones en la placentación como placenta previa y placenta acreta, son las entidades que más contribuyen a la realización de histerectomía obstétrica de emergencia, en los últimos años la histerectomía subtotal parece ser la técnica de elección para realización de histerectomía obstétrica de emergencia ${ }^{14}$.

La histerectomía ha sido durante mucho tiempo el tratamiento primario para la placenta acreta, recientemente algunos autores han propuesto tratamientos conservadores como una alternativa para la preservación de la fertilidad, sin embargo estos estudios son solamente reporte de casos o series muy pequeñas, por lo tanto, no existe consenso en cuanto a cuál procedimiento adoptar ${ }^{15}$.

En esta era de incremento acelerado de la cesárea, se debe hacer énfasis en realizar un correcto y precoz diagnóstico prenatal de los defectos de la implantación placentaria para realizar una remisión a un nivel de atención adecuado, realizar un enfoque multidisciplinario y prevenir la morbilidad materna ante la posibilidad de presentarse un aborto, signos sugestivos de placenta acreta pueden presentarse 
tempranamente durante el primer trimestre. Algunas de las imágenes recomendadas en la evaluación de la invasión placentaria incluyen la ultrasonografía Doppler, ultrasonografía con escala de grises y la resonancia magnética $(\mathrm{RM})^{11,16}$. En general la ultrasonografía con escala de grises predice placentación anormal con una sensibilidad de $77 \%$ a $86 \%$ y una especificidad del $96 \%$ a $98 \%$, para la ultrasonografía Doppler y Doppler color la sensibilidad aumenta al 97\% con una especificidad de $92 \%{ }^{11,17}$.

Los siguientes son hallazgos sugestivos de placenta Acreta:

- Presencia de múltiples lagos placentarios.

- Desaparición del espacio retroplacentario miometral libre.

- Afinamiento o desaparición de la capa miometral.

- Disrupción o irregularidad del plano de clivaje uterinovesical.

- Tejido placentario con efecto de masa (abombamiento).

- Irregularidad de la pared vesical.

- Interrupción de la interface pared posterior de la vejiga-pared uterina.

- Obliteración del espacio claro entre el útero y la placenta.

- Hipervascularización de la pared vesical subyacente.

- Grosor miometrial más delgado menor a $1 \mathrm{~mm}$.

Muchos autores han encontrado similitudes entre la ultrasonografía y la RM para diagnosticar placenta acreta, sin embargo el alto costo de la RM y el nivel de experticia requerida para una adecuada interpretación hace que solamente sea recomendada para casos ambiguos. Se ha reportado la RM para diagnóstico de placenta acreta con una sensibilidad entre $78 \%$ a $88 \%$ con especificidad entre el $67 \%$ a $100 \%{ }^{11,18}$. La cistoscopia puede ser usada para detectar compromiso vesical cuando existe una fuerte sospecha de placenta percreta.

\section{Conclusiones}

La placenta acreta es una de las complicaciones obstétricas más temidas, pudiendo derivar de ellas otro tipo de complicaciones graves, el manejo de esta entidad debe ser realizado en un nivel hospitalario terciario y un equipo multidisciplinario, mejorando de esta forma los resultados para la paciente. Existen pocos casos de acretismo placentario en abortos tardíos, se debe tener por parte del Obstetra un alto índice de sospecha en aquellas pacientes que presenten factores de riesgo para desarrollar esta entidad y de esta manera impactar positivamente en la morbilidad y mortalidad.

\section{Referencias}

1. Publications Committee, Society for Maternal-Fetal Medicine, Belfort MA. Placenta accreta. Am J Obstet Gynecol. 2010;203:430-439.

2. Wong HS, Cheung YK, Zuccollo J, et al. Evaluation of sonographic diagnostic criteria for placenta accrete. J Clin Ultrasound. 2008;36:551-9.
3. Garmi G, Salim R. Epidemiology, etiology, diagnosis, and management of placenta accreta. Obstet Gynecol Int. 2012;2012:87392-9.

4. Eshkoli T, Weintraub AY, Sergienko R, et al. Placenta accreta: risk factors, perinatal outcomes, and consequences for subsequent births. Am J Obstet Gynecol. 2013;208:219.e1-7.

5. Timor-Tritsch I, Monteagudo A. Unforeseen consequences of the increasing rate of cesarean deliveries: early placenta accrete and cesarean scar pregnancy. A review. Am J Obstet Gynecol. 2012; 14-29.

6. Esh-Broder E, Ariel I, Abas-Bashir N, et al. Placenta accreta is associated with IVF pregnancies: a retrospective chart review. BJOG. 2011;118:1084-9.

7. Jauniaux E, Jurcovik D. Placenta accreta: Pathogenesis of a 20th century of iatrogenic uterine disease. Placenta. 2012;33:244-251.

8. MacDorman MF, Menacker F, Declercq E. Cesarean birth in the United States: epidemiology, trends and outcomes. Clin Perinatol. 2008;35(2):293-307.

9. Hamilton BE, Martin JA, Ventura SJ. Births: preliminary data for 2010. Natl Vital Stat Rep. 2011;60(2):1-26.

10. Silver RM, Landon MB, Rouse DJ, et al. Maternal morbidity associated with multiple repeat cesarean deliveries. Obstet Gynecol. 2006;107(6):1226-32.

11. Wortman AC, Alexander JM. Placenta Accreta, Increta and Percreta. Obstet Gynecol Clin N Am. 2013(40): 137 154.

12. Miller DA, Chollet JA, Goodwin TM. Clinical risk factors for placenta previa-placenta accrete. Am J Obtet Gynecol. $1997 ; 177(1): 210-4$.

13. Rashbaum WK, Gates EJ, Jones J, et al. Placenta accreta encountered during dilation and evacuation in the second trimester. Obstetrics and Gynecology. 1995;85:701-703.

14. Lone F, Sultan AH, Thakar R, et al. Risk factors and management patterns for emergency obstetric hysterectomy over 2 decades. International Journal of Gynecology and Obstetrics. 2010;109:12-15.

15. Bretelle $F$, Courbière $B$, Mazouni $C$, et al. Management of placenta accreta: morbidity and outcome. European Journal of Obstetrics and Gynecology and Reproductive Biology. 2007;133:34-39.

16. Mazouni C, Gorincour G, Juhan V, et al. Placenta accreta: a review of current advances in prenatal diagnosis. Placenta. 2007;28:599-603.

17. Shih JC, Palacios Jaraquemada JM, Su YN, et al. Role of three-dimensional power Doppler in the antenatal diagnosis of placenta accreta: comparison with grayscale and color Doppler techniques. Ultrasound in Obstetrics \& Gynecology. 2009;33:193-203.

18. Warshak CR, Eskander R, Hull AD, et al. Accuracy of ultrasonography and magnetic resonance imaging in the diagnosis of placenta accrete. Obstetrics \& Gynecology. 2006;108:573-81. 